\title{
Maximum likelihood-based adaptive iteration algorithm design for joint CFO and channel estimation in MIMO-OFDM systems
}

Nan-Hung Cheng ${ }^{1 *}$, Kai-Chieh Huang ${ }^{1}$, Yung-Fang Chen ${ }^{1}$ and Shu-Ming Tseng ${ }^{2}$

* Correspondence: cheng-wind@ yahoo.com.tw

${ }^{1}$ Department of Communication Engineering, National Central University, Jungli City, Taoyuan, Taiwan, Republic of China

Full list of author information is available at the end of the article

\begin{abstract}
In this paper, we present a joint time-variant carrier frequency offset (CFO) and frequency-selective channel response estimation scheme for multiple input-multiple output-orthogonal frequency-division multiplexing (MIMO-OFDM) systems for mobile users. The signal model of the MIMO-OFDM system is introduced, and the joint estimator is derived according to the maximum likelihood criterion. The proposed algorithm can be separated into three major parts. In the first part of the proposed algorithm, an initial CFO is estimated using derotation, and the result is used to apply a frequency-domain equalizer. In the second part, an iterative method is employed to locate the fine frequency peak for better CFO estimation. An adaptive process is used in the third part of the proposed algorithm to obtain the updated CFO estimation and track parameter time variations, including the time-varying CFOs and time-varying channels. The computational complexity of the proposed algorithm is considerably lower than that of the maximum likelihood-based grid search method. In a simulation, the mean squared error performance of the proposed algorithm was close to the Cramer-Rao lower bound. The simulation results indicate that the proposed novel joint estimation algorithm provides a bit error rate performance close to that in the perfect channel estimation condition. The results also suggest that the proposed method has reliable tracking performance in Jakes' channel models.
\end{abstract}

Keyword: Joint maximum likelihood estimation, Carrier frequency offset (CFO), Multiple input multiple output (MIMO), Orthogonal frequency-division multiplexing (OFDM)

\section{Introduction}

Many researchers regard multiple input multiple output (MIMO) as a candidate technology for fifth-generation mobile networks (5G). With spatial multiplexing, antennas transmit (TX) independent data streams simultaneously to improve the system throughput $[1,2]$. With orthogonal frequency-division multiplexing (OFDM), overlapping but orthogonal subchannels are introduced to convert a frequency-selective fading

(c) The Author(s). 2021 Open Access This article is licensed under a Creative Commons Attribution 4.0 International License, which permits use, sharing, adaptation, distribution and reproduction in any medium or format, as long as you give appropriate credit to the original author(s) and the source, provide a link to the Creative Commons licence, and indicate if changes were made. The images or other third party material in this article are included in the article's Creative Commons licence, unless indicated otherwise in a credit line to the material. If material is not included in the article's Creative Commons licence and your intended use is not permitted by statutory regulation or exceeds the permitted use, you will need to obtain permission directly from the copyright holder. To view a copy of this licence, visit http://creativecommons.org/licenses/by/4.0/. 
channel into a set of frequency-flat fading channels for easier equalization [3]. From another perspective, OFDM can resist inter-symbol interference (ISI) by appending cyclic prefix $(\mathrm{CP})$ samples in front of OFDM samples.

In the case of single-antenna OFDM systems or other multicarrier systems, three aspects should generally be managed: frequency synchronization, timing synchronization, and channel estimation. Multiple input-multiple output-orthogonal frequency-division multiplexing (MIMO-OFDM) is extremely sensitive to frequency synchronization and channel estimation errors [4]. Carrier frequency offsets (CFOs) are induced by local oscillator mismatches between transmitters and receivers as well as Doppler shifts [5]. CFOs break subcarrier orthogonality, which results in intercarrier interference and the possible performance degradation of the system [6]. Compared with frequency synchronization and channel estimation, timing synchronization is less critical because $\mathrm{CP}$ insertion has some tolerance to timing errors [7]. In this paper, we assume the timing offsets are equal to zero and focus on frequency synchronization and channel estimation.

To achieve high transmission quality, a frequency offset must be estimated and compensated at the receiver [8]. The combined estimation of the channel and the frequency offset causes complex problems in MIMO-OFDM systems due to the number of unknown parameters. In some estimation algorithms, CFO and channel estimations are treated separately by using different training sequences [9-12]. In these schemes, channel estimation is performed assuming zero CFO or frequency synchronization is achieved. However, such assumptions are rarely valid in practice with the presence of noise $[9,10]$.

In other schemes, to save bandwidth, joint estimation of the channel and frequency offset is attempted. A prohibitively high computational complexity is required to obtain maximum likelihood (ML) solutions for both the frequency offset and channel impulse response (CIR) [13]. These estimators in [3, 5-7, 9-13] are designed for single-input single-output OFDM systems and not MIMO-OFDM systems. In [14, 15], the channel estimation of MIMO-OFDM systems was performed assuming no CFO imbalance or perfect frequency synchronization with the training sequences. In $[16,17]$, the proposed algorithms for CFO estimation were executed assuming an estimated channel and negligible channel effect. In [18], a method based on the alternating projection algorithm was proposed for ML synchronization and channel estimation in orthogonal frequency-division multiple access uplink transmission. In [19-23], joint estimation algorithms have been proposed for time-frequency synchronization with channel identification in MIMO-OFDM systems. However, all these algorithms have rather high complexity. In [24-26], an iterative method was used to reduce the complexity. However, in these schemes in [24-26], the CFO estimator must use point search, which may assume a search range with a small interval to achieve low complexity. Moreover, the methods presented in [18-26] do not provide the ability to track time-varying CFOs and time-varying channels simultaneously.

This paper discusses the problem of joint CFO and channel estimation in MIMOOFDM systems for mobile users, in which all subcarriers can be utilized simultaneously. The complexity of joint ML estimation through a grid search procedure motivated us to develop a new iteration algorithm [27] that can provide ML solutions for joint estimation problems in an iterative manner. The proposed algorithm can find 
the initial estimated CFO by employing a derotation algorithm and use this result as an initial value. It then uses the initial value to apply the frequency-domain equalizer and uses the derotation operation again for better estimation. Subsequently, an iterative method is adopted to improve estimation accuracy. To benchmark the performance of the proposed scheme, Cramer-Rao lower bounds (CRBs) were derived for the CFO and the CIR. Finally, the CFO was estimated through a proposed parameterized adaptive process by tracking channels and CFOs simultaneously. The simulation results indicated the suitable performance of the proposed adaptive iteration estimator. The computational complexity of the proposed algorithm is lower than that of the grid search-based method. Our contribution and new ideas are as follows: (a) a joint CFO and channel estimation in MIMO-OFDM systems for mobile users, (b) a new iteration algorithm with lower complexity than the grid search-based method, and (c) a newly designed mechanism with an adaptive mode to simultaneously track the time-varying CFOs and time-varying channels. Up to the present time, the proposed adaptive iteration algorithm is currently a state-of-the art approach for achieving near-optimal performance for this joint CFO and channel estimation problem. To the best of our knowledge, in other studies such as [18-26], no report has investigated the joint CFO and channel estimation problem with simultaneous tracking of the time-varying CFOs and time-varying channels.

The remainder of this paper is organized as follows. Section 2 presents the system model. Section 3 describes the development of the adaptive iterative CFO estimation algorithm. Section 4 presents the simulation results, which indicate that the performance of the proposed algorithm is close to the CRBs. Finally, Section 5 reports the conclusions.

Notation: The superscripts $(\cdot)^{T},(\cdot)^{-1},(\cdot)^{H}$, and $\operatorname{diag}(\cdot)_{K \times K}$ represent the transpose, inverse, Hermitian transpose, and $K \times K$ diagonal matrix, respectively. The $K \times K$ identity matrix is denoted by $\mathbf{I}_{K \times K}$.

\section{System model}

Consider a MIMO-OFDM communication system with $K$ subcarriers as well as $N$ transmitting (TX) and $M$ receiving (RX) antennas (Fig. 1).

The model of each transmitter structure of the MIMO-OFDM system is illustrated in Fig. 2 [28].

At the $j$ th transmitter, the preamble OFDM signal in the baseband time domain obtained after performing inverse discrete Fourier transform and CP insertion can be denoted as follows [29]:

$$
x_{j}(k)=\left\{\begin{array}{lr}
\frac{1}{\sqrt{K}} \sum_{k=-N_{g}}^{K-1} d_{j}(k) e^{j 2 \pi n k / K}, \text { if }-N_{g} \leq k \leq K-1 \\
0, & \text { otherwise }
\end{array}\right.
$$

where $d(k)$ is the frequency-domain data symbol for each subcarrier $(k=0,1, \ldots, K)$ and $N_{g}$ represents the CP samples of the OFDM symbol, which is used for ISI resistance. The discrete-time composite CIR between the $j$ th transmitter and the $i$ th received antenna with $L$ multipaths is represented as follows:

$$
\mathbf{h}_{i, j}=\left[h_{i, j}(0), h_{i, j}(1), \ldots, h_{i, j}(L-1)\right]^{\mathrm{T}}, \quad 1 \leq i \leq M, 1 \leq j \leq N
$$



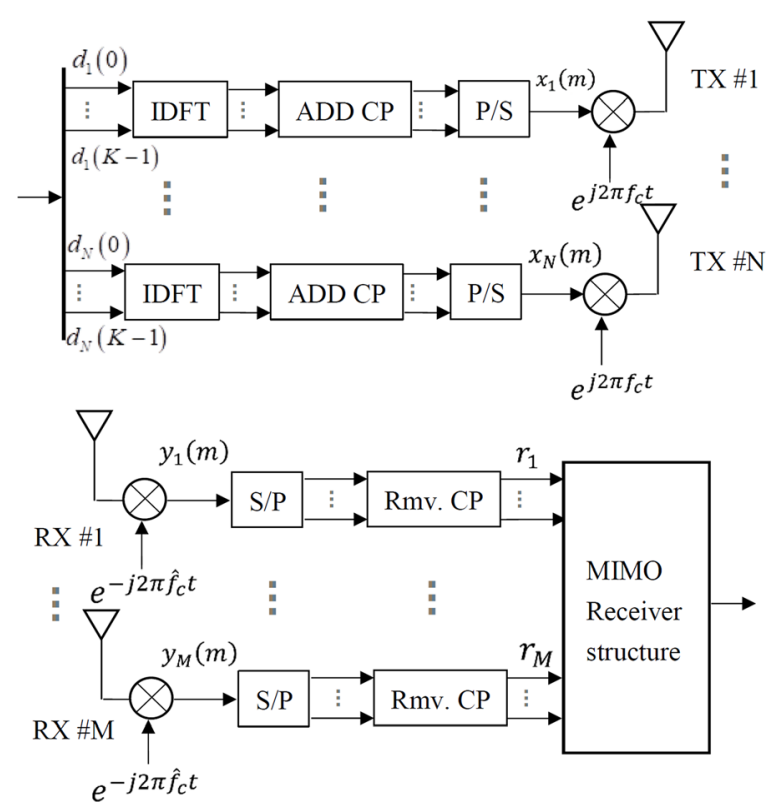

Fig. 1 Transmitter and receiver structure of $N \times M$ MIMO-OFDM system

where $h_{i, j}(l), l=0,1, \ldots, L-1$ is the complex Gaussian gain of the $l$ th multipath. We assume the complex CIR is time-invariant over one OFDM symbol. Thus, the superposition of signals from all TX antennas plus noise is received at each RX antenna. Assume that all the received signals are down-converted to baseband with the same local oscillator centered at $f_{c}$. In the presence of CFO $\varepsilon$, the samples at the $i$ th $\mathrm{RX}$ antenna can be represented as follows:

$$
y_{i}(m)=e^{j(2 \pi \varepsilon / K) m} \sum_{j=1}^{N} \sum_{l=0}^{L-1} h_{i, j}(l) \cdot x_{j}(m-l)+v_{i}(m), i=1, \ldots, M
$$

where $v_{i}(m)$ is an additive white complex Gaussian distributed noise with a mean of 0 and variance of $\sigma_{v_{i}}^{2}$. Moreover, $\varepsilon$ denotes the CFO normalized to the subcarrier spacing [5]. To avoid ISI, we assume that the timing offset is equal to 0 and that the number of multipaths $(L)$ is smaller than the CP length.

According to (3), the received signal vector $\mathbf{r}_{i}=\left[y_{i}(0), y_{i}(1), \ldots, y_{i}(K-1)\right]^{\mathrm{T}}$ at the $i^{\text {th }} \mathrm{RX}$ antenna can be used to rewrite the input-output relationship as follows:

$$
\mathbf{r}_{i}=\mathbf{D}(\varepsilon) \sum_{j=1}^{N} \mathbf{X}_{j} \mathbf{h}_{i, j}+\mathbf{v}_{i}=\mathbf{D}(\varepsilon) \mathbf{X} \mathbf{h}_{i}+\mathbf{v}_{i}, \quad i=1, \ldots, M
$$

where $\mathbf{D}(\varepsilon)=\operatorname{diag}\left(1, e^{j 2 \pi \varepsilon / K}, \ldots, e^{j 2 \pi(K-1) \varepsilon / K}\right)_{K \times K}$ is a diagonal CFO matrix, $\mathbf{X}_{j}$ is a $K \times L$

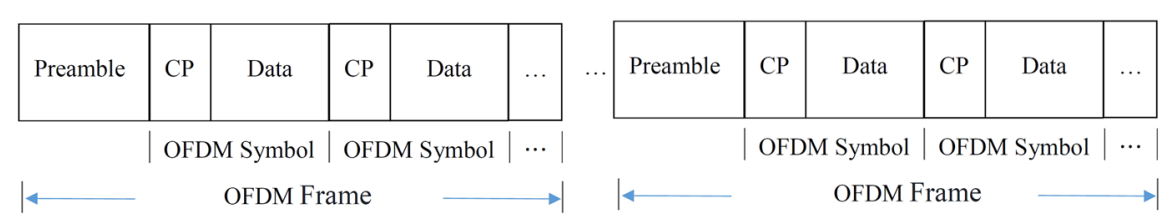

Fig. 2 Each transmitter structure of preamble MIMO-OFDM system 
circular matrix formed by the $j^{t h}$ transmitted signal $x_{j}(k)$, and $\mathbf{v}_{i}$ is a $K \times 1$ noise vector that can be expressed using the covariance matrix $\sigma_{v_{i}}^{2} \mathbf{I}_{K \times K}$. Then, we define two matrices $\mathbf{X}$ and $\mathbf{h}_{i}$ as follows:

$$
\mathbf{X}_{j}=\left[\begin{array}{cccc}
x_{j}(0) & x_{j}(K-1) & \cdots & x_{j}(1-L) \\
x_{j}(1) & x_{j}(0) & \cdots & x_{j}(2-L) \\
x_{j}(2) & x_{j}(1) & \cdots & x_{j}(3-L) \\
\vdots & \vdots & \ddots & \vdots \\
x_{j}(K-1) & x_{j}(K-2) & \cdots & x_{j}(K-L)
\end{array}\right]_{K \times L} \quad, j=1, \ldots, N \boldsymbol{X}=\left[\begin{array}{llll}
X_{1} & \cdots & \boldsymbol{X}_{N}
\end{array}\right]_{K \times N \cdot L} ;
$$

Let $\mathbf{D}(\varepsilon) \mathbf{X h} \mathbf{h}_{i}=\mathbf{s}_{i}$ and $\mathbf{s}_{i}=\left[s_{i}(0), \ldots, s_{i}(K-1)\right]^{T}$ so that the signal-to-noise ratio (SNR) is defined [10] as follows:

$$
\mathrm{SNR}=\sigma_{s_{i}}^{2} / \sigma_{v_{i}}^{2}
$$

where

$$
\sigma_{\mathbf{s}_{i}}^{2}=\frac{1}{K} \sum_{k=0}^{K-1}\left|s_{i}(k)\right|^{2} \quad, \mathrm{k}=0,1, \ldots, K-1 .
$$

\section{Proposed joint CFO and channel iterative estimation algorithm}

\subsection{Receiver design}

The processing diagram at the receiver for the proposed algorithm is displayed in Fig. 3.

In the common RX structure used in OFDM transmission, a frequency-domain equalizer is adopted to eliminate multipath interference. The coefficients of the equalizer are the results obtained through channel estimation. These results are fed back to the time-domain signal, and the steps are run again to obtain more accurate CFO estimates. The main idea is that the proposed algorithm combines the CFOs estimated in the previous processes as a rough estimated value for the followed iteration. Through small-step iterative searching, we can find the maximum peak near the actual $\mathrm{CFO}$ as the fine-tuned estimate. Finally, the CFO adaptation process is performed using the final fine-tuned CFO estimate to improve the performance of the proposed estimator for tracking the time-varying parameters. The aforementioned are described in detail as the following text.

\subsection{Initial CFO estimation}

According to (4) and the Gaussian probability density function, the ML function can be written as follows:

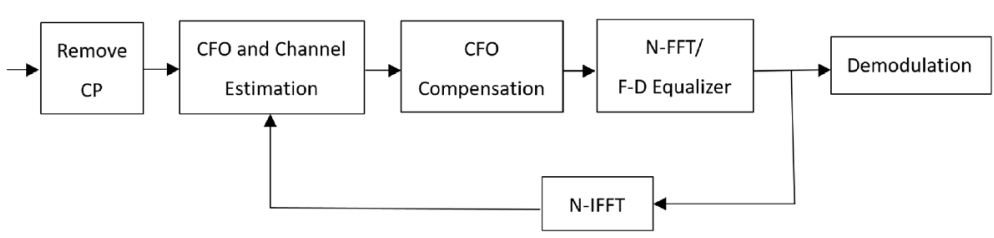

Fig. 3 Processing diagram of the proposed MIMO-OFDM receiver structure 


$$
\Lambda\left(\mathbf{h}_{i}, \varepsilon\right)=\frac{1}{\left(\pi \sigma_{v_{i}}^{2}\right)^{K}} e^{-\frac{1}{\sigma_{v_{i}}^{2}\left\|\mathbf{r}_{i}-\mathbf{D}(\varepsilon) \mathbf{X} \mathbf{h}_{i}\right\|^{2}}} .
$$

Then, the log-likelihood function for two unknown parameters can be expressed as follows:

$$
L_{i}\left(\varepsilon, \mathbf{h}_{i}\right)=-K \ln \left(\pi \sigma_{\mathbf{v}_{i}}^{2}\right)-\frac{1}{\sigma_{\mathbf{v}_{i}}^{2}}\left\|\mathbf{r}_{i}-\mathbf{D}(\varepsilon) \mathbf{X} \mathbf{h}_{i}\right\|^{2} .
$$

To evaluate the log-likelihood function in (9), the constant is eliminated to minimize the newly defined objective function, which is the second term of the log-likelihood function.

$$
\phi\left(\varepsilon, \mathbf{h}_{i}\right) \stackrel{\Delta}{=}\left\|\mathbf{r}_{i}-\mathbf{D}(\varepsilon) \mathbf{X \mathbf { h } _ { i }}\right\|^{2}=\left(\mathbf{r}_{i}-\mathbf{D}(\varepsilon) \mathbf{X \mathbf { h } _ { i }}\right)^{H}\left(\mathbf{r}_{i}-\mathbf{D}(\varepsilon) \mathbf{X \mathbf { h } _ { i }}\right) .
$$

We can expand (10) and take the partial differentiation operator for $\mathbf{h}_{i}$; then, let the first-order derivative be equal to 0 to obtain $\tilde{\mathbf{h}}_{i}$ as follows:

$$
\tilde{\mathbf{h}}_{i}=\left(\mathbf{X}^{H} \mathbf{X}\right)^{-1} \mathbf{X}^{H} \mathbf{D}^{H}(\varepsilon) \mathbf{r}_{i} .
$$

By substituting (11) into (10), for the purpose of minimizing the ML objective function (10), it becomes to maximize

$$
\bar{\phi}_{1}(\varepsilon)=\mathbf{r}_{i}^{H} \mathbf{D}(\varepsilon) \mathbf{X}\left(\mathbf{X}^{H} \mathbf{X}\right)^{-1} \mathbf{X}^{H} \mathbf{D}^{H}(\varepsilon) \mathbf{r}_{i} .
$$

The estimation of $\varepsilon$ for the $i^{t h} \mathrm{RX}$ antenna can be obtained as follows:

$$
\hat{\varepsilon}_{i}=\underset{\varepsilon}{\arg }\left\{\max \left[\mathbf{r}_{i}^{H} \mathbf{D}(\varepsilon) \mathbf{X}\left(\mathbf{X}^{H} \mathbf{X}\right)^{-1} \mathbf{X}^{H} \mathbf{D}^{H}(\varepsilon) \mathbf{r}_{i}\right]\right\} .
$$

Equation (13) can be used to perform a grid search to find the optimal CFO. However, this process requires many computations and is thus difficult to implement. The projection matrix $\mathbf{X}\left(\mathbf{X}^{H} \mathbf{X}\right)^{-1} \mathbf{X}^{H}$ is formed by the preamble signal per frame transmission. The projection matrix can be represented as follows:

$$
\left[\begin{array}{ccccc}
\mathbf{X}^{H} \mathbf{X}= & & & & \\
R_{1} & C_{1,2} & \ldots & C_{1, N \cdot L-1} & C_{1, N \cdot L} \\
C_{2,1} & R_{2} & \ldots & C_{2, N \cdot L-1} & C_{2, N \cdot L} \\
\vdots & \vdots & \ddots & \vdots & \vdots \\
C_{N \cdot L-1,1} & C_{N \cdot L-1,2} & \ldots & R_{N \cdot L-1} & C_{N \cdot L-1, N \cdot L} \\
C_{N \cdot L, 1} & C_{N \cdot L, 2} & \ldots & C_{N \cdot L, N \cdot L-1} & R_{N \cdot L}
\end{array}\right]_{N \cdot L \times N \cdot L}
$$

The main diagonals of the $\mathbf{X}^{H} \mathbf{X}$ matrix are real-valued and are denoted as $R_{i}, i=1, \ldots$, $N \cdot L$. Its adjacent diagonals parallel to the main diagonals are denoted as $C_{i, j}, i=1, \ldots$, $N \cdot L, j=1, \ldots, N \cdot L, i \neq j$, which represent conjugate symmetry on the other side parallel to the main diagonal. The matrix $\mathbf{X}\left(\mathbf{X}^{H} \mathbf{X}\right)^{-1} \mathbf{X}^{H}$ has a similar structure.

$$
\begin{aligned}
& \mathbf{X}\left(\mathbf{X}^{H} \mathbf{X}\right)^{-1} \mathbf{X}^{H}= \\
& {\left[\begin{array}{ccccc}
W_{1} & E_{1,2} & \cdots & E_{1, K-1} & E_{1, K} \\
E_{2,1} & W_{2} & \ldots & E_{2, K-1} & E_{2, K} \\
\vdots & \vdots & \ddots & \vdots & \vdots \\
E_{K-1,1} & E_{K-1,2} & \cdots & W_{K-1} & E_{K-1, K} \\
E_{K, 1} & E_{K, 2} & \cdots & E_{K, K-1} & W_{K}
\end{array}\right]_{K \times K}}
\end{aligned}
$$


where $W_{i}, i=1, \ldots, K$, are real-valued and $E_{i, j}, i=1, \ldots, K, j=1, \ldots, K, i \neq j$, are complex valued and are the conjugate of $E_{j, i}$.

By substituting (15) into (13), the estimation of $\varepsilon$ for the $i^{\text {th }} \mathrm{RX}$ antenna can be expressed as follows:

$$
\begin{aligned}
& \hat{\varepsilon}_{i}=\underset{\varepsilon}{\arg }\left\{\max \left[\mathbf{r}_{i}^{H} \mathbf{D}(\varepsilon) \mathbf{X}\left(\mathbf{X}^{H} \mathbf{X}\right)^{-1} \mathbf{X}^{H} \mathbf{D}^{H}(\varepsilon) \mathbf{r}_{i}\right]\right\} \\
& =\underset{\varepsilon}{\arg \max }\left\{\begin{array}{l}
\sum_{k=1}^{K-1} r_{i}^{2}(k) W_{k+1}+ \\
2 \operatorname{Re}\left[\begin{array}{l}
\sum_{k=0}^{K-2} r_{i}^{*}(k+1) r_{i}(k) e^{j 2 \pi \varepsilon / K} E_{k+2, k+1}+ \\
K-3 \\
k=0 \\
\sum_{k=0}^{1} r_{i}^{*}(K-2+k) r_{i}(k) e^{j 2 \pi(2) \varepsilon / K} E_{k+3, k+1}+\cdots+ \\
+r_{i}^{*}(K-1) r_{i}(0) e^{j 2 \pi(K-1) \varepsilon / K} E_{K, 1} e^{j 2 \pi(K-2) \varepsilon / K} E_{K-1+k, k+1}
\end{array}\right]
\end{array}\right\} .
\end{aligned}
$$

To find the maximum value for the estimation of $\varepsilon$, (16) can be separated into multiple terms. Because the first term in (16) is a fixed real value, we only consider the second part. In the second part, because the first term is a complex value caused by the frequency offset, we can derotate its phase to the real axis; thus, the frequency offset can be compensated to achieve a maximum real value. The frequency offsets of $K$ terms for the $i$ th $\mathrm{RX}$ antenna can be obtained as follows:

$$
\begin{gathered}
\tilde{\varepsilon}_{i}(k)=\operatorname{angle}\left(\sum_{a=0}^{K-k-1} E_{a+k+1, a+1} r_{i}^{*}(a+k) r_{i}(a)\right) K /(2 k \pi), \\
k=1, \ldots, K-1 .
\end{gathered}
$$

After finding the phase of each term of (17) associated with the maximum real value, we can average the phases of all the terms for each RX antenna and then use the calculated average values to obtain the initial CFO as follows:

$$
\tilde{\varepsilon}=\frac{1}{M} \sum_{i=1}^{M} \frac{1}{(K-1)} \sum_{k=1}^{K-1} \tilde{\varepsilon}_{i}(k) .
$$

\subsection{Frequency-domain equalizer}

The initial CFO performance degrades due to multipath interference and may not be accurate. Therefore, a frequency-domain equalizer is applied to overcome this problem and obtain a more accurate CFO. First, we utilize the initial CFO (18) to find the channel response (11). Second, we use the initial CFO to compensate the received signal, which is then transformed into the frequency domain. A frequency-domain equalizer is employed using the estimated channel response to cancel the multipath interference [30]. The aforementioned operations are expressed as follows: 


$$
\tilde{\mathbf{y}}_{i}=(\mathbf{D}(\tilde{\varepsilon}))^{\mathrm{H}} \mathbf{r}_{i}=(\mathbf{D}(\tilde{\varepsilon}))^{\mathrm{H}}\left(\mathbf{D}(\varepsilon) \sum_{j=1}^{N} \mathbf{X}_{j} \mathbf{h}_{i, j}+\mathbf{v}_{i}\right) .
$$

If $\tilde{\varepsilon}=\varepsilon$, we can rewrite the received signal (19) and transform it into the frequency domain as follows:

$$
\tilde{\mathbf{Y}}_{i}=\mathbf{F} \tilde{\mathbf{y}}_{i}=\sum_{j=1}^{N} \mathbf{H}_{i, j} \mathbf{s}_{j}+\mathbf{v}_{i}^{\prime}
$$

where $\mathbf{H}_{i, j}$ is a diagonal matrix that is the frequency-domain channel matrix. The term $\mathbf{H}_{i, j}=\operatorname{diag}\left(\overline{\mathbf{h}}_{i, j}\right), \overline{\mathbf{h}}_{i, j}=\left[\bar{h}_{i, j}(0), \bar{h}_{i, j}(1), \ldots, \bar{h}_{i, j}(K-1)\right]^{\mathrm{H}}$ represents a $K \times 1$ vector that includes the samples from the $K$-point discrete Fourier transform of the channel response, and $\mathbf{s}_{j}$ is the frequency-domain signal of the $j$ th TX antennas. We can rewrite the signal received at all antennas over the $k$ th subcarrier as follows:

$$
\begin{aligned}
& \tilde{\boldsymbol{\gamma}}_{k}=\left[\tilde{\boldsymbol{\gamma}}_{1}(k), \tilde{\boldsymbol{\gamma}}_{2}(k), \ldots, \tilde{\boldsymbol{\gamma}}_{M}(k)\right]_{M \times 1}^{\mathrm{T}} \\
& \tilde{\boldsymbol{\gamma}}_{k}=\tilde{\mathbf{H}}_{k} \tilde{\mathbf{s}}_{k}+\mathbf{v}_{k}, \quad k=0,1, \ldots, K-1
\end{aligned}
$$

where $\tilde{\mathbf{H}}_{k} \in \mathbb{C}^{M \times N}$ is the MIMO channel matrix over the $k$ th subcarrier and $\tilde{\mathbf{s}}_{k} \in \mathbb{C}^{N \times 1}$ is the signal transmitted over the $k$ th subcarrier. According to the signal at each subcarrier, we can equalize the compensated received signals as follows:

$$
\widehat{\widetilde{\mathbf{s}}}_{k}=\left(\tilde{\mathbf{H}}_{k}^{H} \tilde{\mathbf{H}}_{k}\right)^{-1} \tilde{\mathbf{H}}_{k}^{H} \tilde{\gamma}_{k}, \quad k=0,1, \ldots, K-1
$$

Subsequently, we can collect $K$ consecutive samples and transform the equalized signals back into the time domain. The derotation method is then used again to calculate the more accurate CFO $\tilde{\varepsilon}_{e}$.

In the following section, an iterative method is described. This method provides a refined estimate close to the CRB.

\subsection{Small-step iterative searching}

The CFO estimation is refined using an iteration method. The CFO $\tilde{\varepsilon}_{e}$ derived in the previous section is used as the initial main frequency estimate. Then, adjacent frequencies are selected near the initial main frequency as candidate frequencies. The next step is to substitute the main and candidate frequencies into (16) to evaluate the CFO values. The largest CFO function value is determined to identify the search direction (refer to Fig. 4, step 1). Subsequently, the frequency selected in the previous step becomes the new main frequency $\tilde{\varepsilon}_{m}$. The fixed-step frequency $\Delta f$ is added with the new main frequency $\tilde{\varepsilon}_{m}$ to acquire its adjacent frequency, and the comparison is performed as before (refer to Fig. 4, steps $2-1$ and 2-2). This iterative process continues until the function value of the main frequency in the evaluation is larger than that of its adjacent frequency (refer to Fig. 4, steps 2 and 3 ; e.g., the total number of iterations is 3 ). Finally, the maximum frequency peak $\varepsilon_{f}$ is achieved in the region between the last main frequency and the last adjacent frequency (refer to Fig. 4, step 3). 


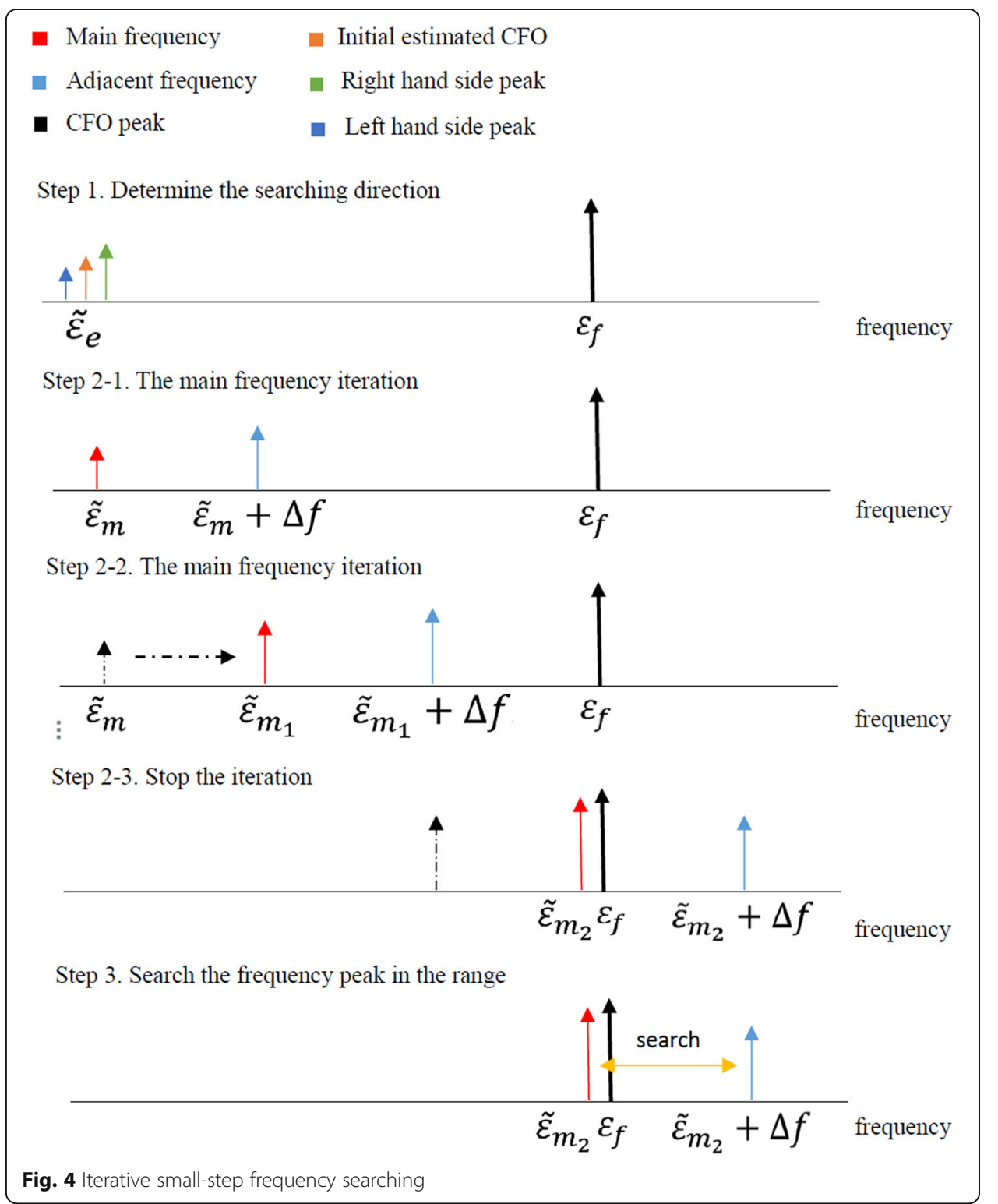

However, the global maximum frequency peak may not be in the search region if the setting of a small-step frequency is inappropriate. For instance, the CFO estimated with the iterative method would not be at the true frequency peak if the true global maximum frequency is in the previous range between the iterative main frequency and the iterative adjacent frequency (refer to Fig. 5, steps 2 and 3). Because the iterative adjacent frequency $\tilde{\varepsilon}_{m_{i}}+\Delta f$ is larger than the iterative main frequency $\tilde{\varepsilon}_{m_{i}}$, the maximum frequency is decided to be located at the right-hand side of the iterative adjacent frequency. Therefore, the iteration continues until the result of the main frequency is larger than that of the adjacent frequency (refer to Fig. 5, steps 2-4). However, the peak of the maximum frequency is outside the final search range.

In the aforementioned circumstance, the method of reverse iterative searching is adopted. When the maximum value is at the boundary frequency, the search direction 


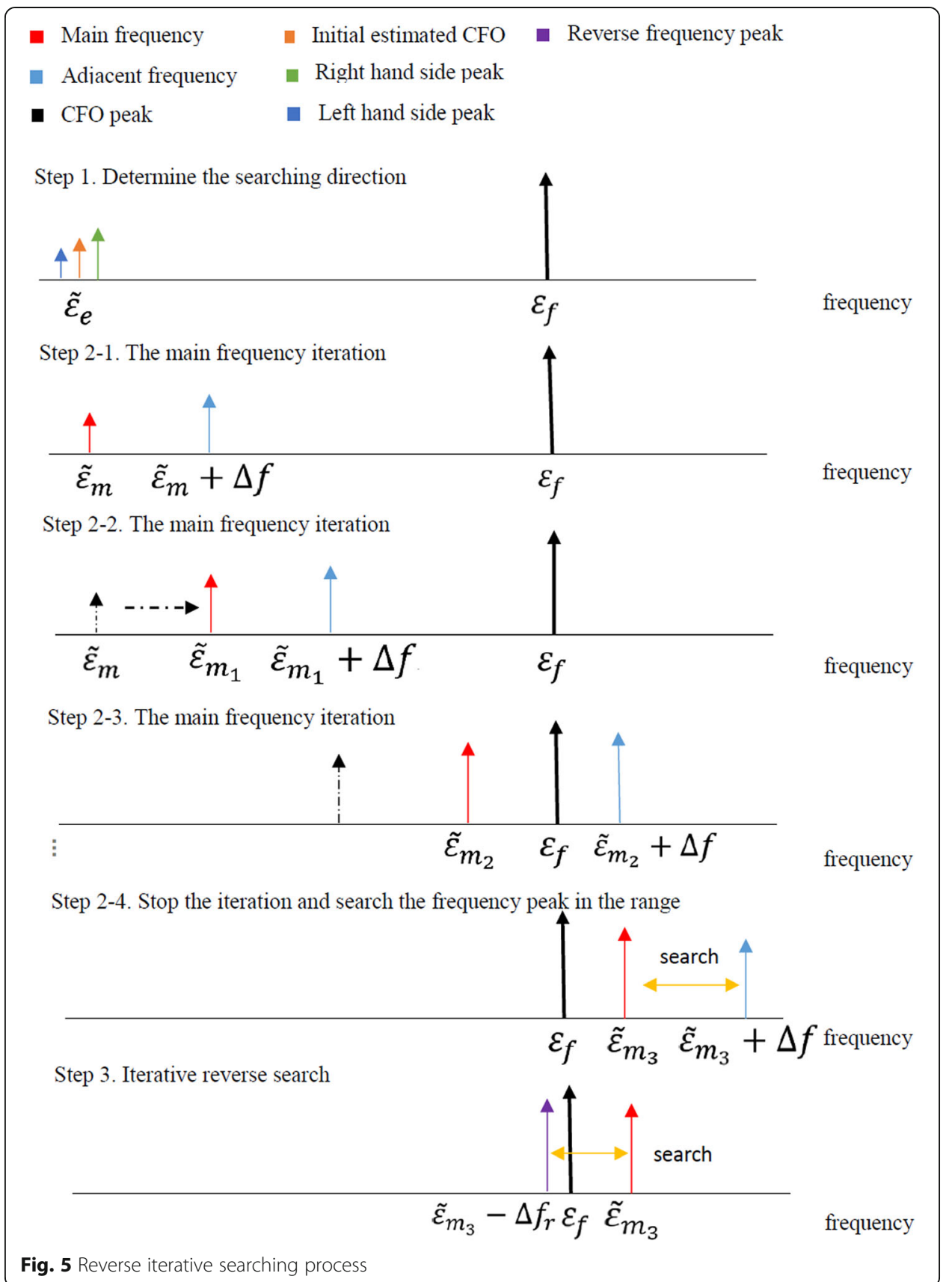

goes in the reverse side (refer Fig. 5, step 3). This process is reverse iterative searching. The maximum peak frequency is on the other side of the boundary frequency. The iterative reverse search method finally identifies the global maximum value (refer to Fig. 5, step 3). Illustrations of the iterative small-step-frequency search process and reverse iterative search process are provided in Figs. 4 and 5, respectively. A large $\Delta f$ can reduce the search time, and a small $\Delta f$ can provide high estimation accuracy. Thus, a trade-off is involved in the selection of the $\Delta f$ value. Practically, an appropriate value is selected based on experimental trial and error results in operational environments and has correlation with SNRs. 
Table 1 Summary of the proposed algorithms

\begin{tabular}{|c|c|}
\hline $\begin{array}{l}\text { Algorithm } \\
\text { procedure }\end{array}$ & Proposed algorithm \\
\hline Step 1 & $\begin{array}{l}\text { Use Eq. (17) to estimate each item of the second part of Eq. (16), and average the results } \\
\text { to obtain the initial estimate of CFO } \tilde{\varepsilon} \text { using Eq. (18). }\end{array}$ \\
\hline Step 2 & $\begin{array}{l}\text { Use the result obtained in step } 1 \text { for channel estimation with Eq. (11) and design the } \\
\text { coefficient of equalizer taps. }\end{array}$ \\
\hline Step 3 & Equalize the received signal by using Eq. (22) and estimate CFO $\tilde{\varepsilon}_{e}$ as in step 1. \\
\hline Step 4 & $\begin{array}{l}\text { Set the main and adjacent frequencies using Eq. (16) from step } 3 \text { and determine the } \\
\text { frequency peak range using the iterative method. }\end{array}$ \\
\hline Step 5 & $\begin{array}{l}\text { Search for the frequency peak } \varepsilon_{f} \text { in the range obtained in step } 4 \text { by inputting the new } \\
\text { main frequency into Eq. (16). }\end{array}$ \\
\hline
\end{tabular}

\subsection{Computational complexity and the procedure of the proposed method}

We adopt the Big-Oh notation, which is a well-accepted approach for analyzing the computational complexities of algorithms. The detailed analysis of the computational complexity is as follows. Note that $K$ is the number of subcarriers and $N$ and $M$ are the numbers of TX and RX antennas, respectively. The proposed scheme comprises five major steps: (1) In step 1, Eqs. (17) and (18) are involved. Taking phase/angle operation may adopt CORDIC IP core, by configuring it to be an arctan mode, which requires a constant time. Therefore, the complexity is assumed to be $O(1)$. The above steps are performed $M K$ times in Eq. (18). Therefore, the computational complexity in step 1 is $O(M K(K+1))=O\left(M K^{2}\right)$. (2) In step 2, Eq. (11) is computed. The term $\left(\mathbf{X}^{H} \mathbf{X}\right)^{-1} \mathbf{X}^{H}$ matrix in Eq. (11) is formed by the preamble signal per frame transmission so that the information of this matrix is treated as already known for both TX and RX. The components of the resultant matrix $\left(\mathbf{X}^{H} \mathbf{X}\right)^{-1} \mathbf{X}^{H}$ can be saved at RX. Therefore, the computational complexity of this matrix $\left(\mathbf{X}^{H} \mathbf{X}\right)^{-1} \mathbf{X}^{H}$ requires $O$ (1) in Eq. (11). Here, we consider the worst-case scenario, where $L$ equals the length of cyclic prefix (CP) samples of the OFDM symbol. The term $\mathbf{D}^{H}(\varepsilon) \mathbf{r}_{i}$ has a computational complexity of $O(K)$. Therefore, the product of $\left(\mathbf{X}^{H} \mathbf{X}\right)^{-1} \mathbf{X}^{H}$ and $\mathbf{D}^{H}(\varepsilon) \mathbf{r}_{i}$ matrices has a computational complexity of $O(K N L)$. The above operations are performed $M$ times in Eq. (11). Therefore, the computational complexity in step 2 is $O(M(K+K N L))$. (3) In step 3, the discrete Fourier transform of the channel response to form $\tilde{\mathbf{H}}_{k}$ by using Fast Fourier Transform, FFT has a computational complexity of $O(M N K \log K)$. The $\tilde{\mathbf{H}}_{k}^{H} \tilde{\mathbf{H}}_{k}$ matrix and its matrix inverse operation have a computational complexity of $O\left(M N^{2}\right)$ and $O$ $\left(N^{3}\right)$, respectively. The calculation of the term $\tilde{\mathbf{H}}_{k}^{H} \tilde{\gamma}_{k}$ has a computational complexity of $O(N M)$. In addition, the matrix $\left(\tilde{\mathbf{H}}_{k}^{H} \tilde{\mathbf{H}}_{k}\right)^{-1} \tilde{\mathbf{H}}_{k}^{H} \tilde{\gamma}_{k}$ has a computational complexity of $O$ $\left(N^{2}\right)$. These operations are performed $K$ times in Eq. (22). Then, the operations in step

Table 2 Summary of the computational complexity analysis

\begin{tabular}{lll}
\hline Algorithm & & Complexity \\
\hline Proposed algorithm & Step 1 & $O\left(M K^{2}\right)$ \\
& Step 2 & $O(M(K+K N L))$ \\
Step 3 & $O\left(M N K \log K+K\left(M N^{2}+N^{3}+N M+N^{2}\right)+M K^{2}\right)$ \\
Steps 4 and 5 & $O\left(M K^{2} \tilde{N}_{\text {iter }}\right)$ \\
$\begin{array}{l}\text { The grid search-based method for CFO estimation without joint } \\
\text { channel estimation }\end{array}$ & $O\left(M K^{2} \tilde{N}_{\text {grid }}\right)$ \\
\hline
\end{tabular}


Table 3 Multipath delay profile

\begin{tabular}{lllll}
\hline & 1st ray & 2nd ray & 3rd ray \\
\hline $\begin{array}{l}\text { Amplitude } \\
\text { and phase }\end{array}$ & $\begin{array}{l}\text { The } \\
\text { iterative } \\
\text { mode }\end{array}$ & $\begin{array}{l}0.81562 \times(\text { random } \\
\text { complex value) }\end{array}$ & $\begin{array}{l}0.49467 \times \text { (random } \\
\text { complex value) }\end{array}$ & $\begin{array}{l}0.300109 \times \text { (random } \\
\text { complex-value) }\end{array}$ \\
$\begin{array}{l}\text { The } \\
\text { adaptive } \\
\text { tracking } \\
\text { mode }\end{array}$ & $\begin{array}{l}0.81562 \times \text { (the coefficients } \\
\text { generated by using the } \\
\text { Jakes' model) }\end{array}$ & $\begin{array}{l}0.49467 \times \text { (the coefficients } \\
\text { generated by using the } \\
\text { Jakes' model) }\end{array}$ & $\begin{array}{l}0.300109 \times \text { (the coefficients } \\
\text { generated by using the } \\
\text { Jakes' model) }\end{array}$ \\
\hline
\end{tabular}

1 are followed with the computational complexity of $O\left(M K^{2}\right)$. Therefore, the computational complexity in step 3 is $O\left(M N K \log K+K\left(M N^{2}+N^{3}+N M+N^{2}\right)+M K^{2}\right)$. (iv) In steps 4 and 5 , the computational complexity of the carrier frequency offset searching process is evaluated by using (16). Let $\tilde{N}_{\text {iter }}$ be the total number of iterations for the convergence of the step frequency search process. The computational complexity in steps 4 and 5 is $O\left(M K^{2} \tilde{N}_{\text {iter }}\right)$. Similarly, let $\tilde{N}_{\text {grid }}$ be the number of the grid search points in the possible CFO range. The total computational complexity of the grid search-based method can be achieved by $O\left(M K^{2} \tilde{N}_{\text {grid }}\right)$. Note that $\tilde{N}_{\text {grid }} \gg K$ and $\tilde{N}_{\text {grid }}$ >> $\tilde{N}_{\text {iter }}$. For instance, if the normalized CFOs are located over $[-0.5,0.5]$, then the number of the grid search-based method in this range would be $\tilde{N}_{\text {grid }}=10^{5}$ if the resolution of $10^{-5}$ is set.

The procedure of the proposed algorithms and the computational complexity are summarized in Tables 1 and 2, respectively.

\subsection{Adaptive mode for tracking the time variations of parameters}

According to the aforementioned described process, the CFO $\tilde{\varepsilon}_{T=t}$ and CFO $\tilde{\varepsilon}_{T=t+\Delta t}$ (CFO of the next time unit) are obtained ( $t$ can be the symbol time, slot duration, frame duration, or any other time unit in the transmission systems). The adjustment equation of the final CFO is updated with the coefficient $\mu$ as follows:

$$
\tilde{\varepsilon}_{e, T=t+\Delta t}=\mu \tilde{\varepsilon}_{T=t}+(1-\mu) \tilde{\varepsilon}_{T=t+\Delta t} .
$$

By substituting (23) into (11), the channel estimation in this adaptive mode is rewritten as follows:

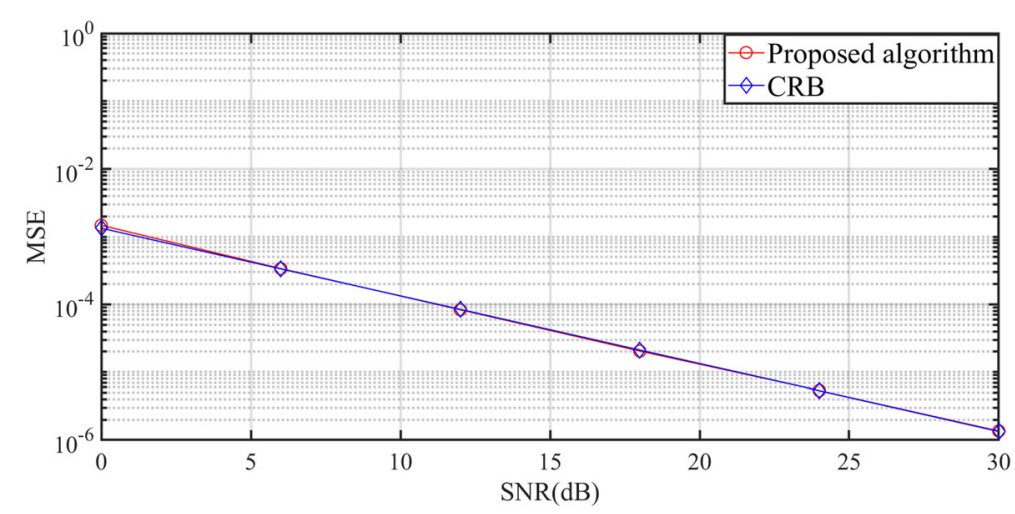

Fig. 6 The MSE performance for CFO estimation of the proposed algorithm compared with CRB 


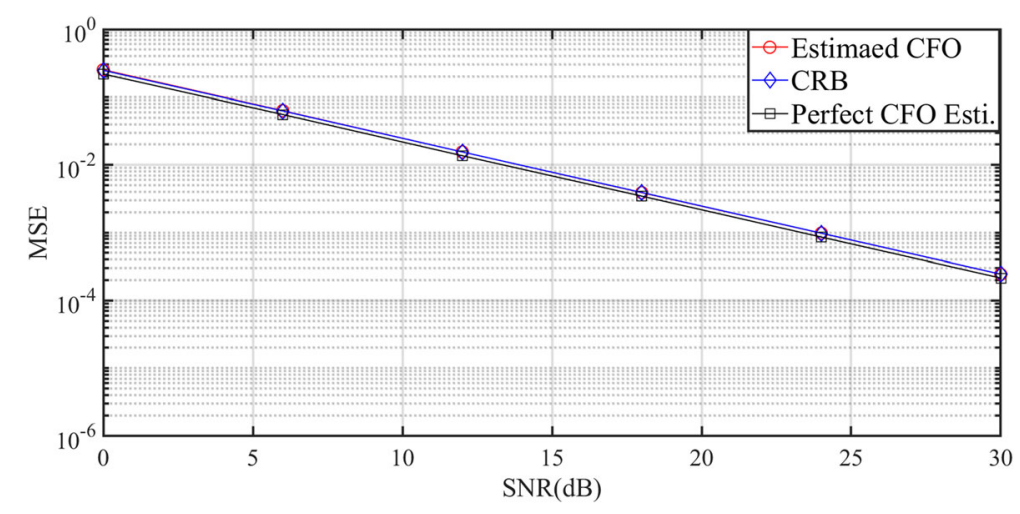

Fig. 7 The MSE performance for channel estimation compared with CRB and the ideal case

$$
\begin{aligned}
& \tilde{\mathbf{h}}_{i, T=t+\Delta t}= \\
& \quad\left(\mathbf{X}_{T=t+\Delta t}^{H} \mathbf{X}_{T=t+\Delta t}\right)^{-1} \mathbf{X}_{T=t+\Delta t}{ }^{H} \mathbf{D}^{H}\left(\tilde{\varepsilon}_{e, T=t+\Delta t}\right) \mathbf{r}_{i, T=t+\Delta t}
\end{aligned}
$$

\section{Simulation}

This section presents the simulation results and discusses the efficacy of the proposed schemes, including the iterative mode and adaptive tracking mode. The parameters common to both the iterative mode and adaptive tracking mode were as follows: $M=2$, $N=2, K=64$, and the CP size $N_{g}=16$. A preamble was inserted at the beginning of each transmitted signal per frame. The transmitted OFDM data symbols were modulated through quaternary phase-shift keying by using a three-multipath channel model, and estimations were made on the basis of the preamble. The delay profile for the multipaths is presented in Table 3. The channel gain of each multipath was randomly generated with a Gaussian distribution of variance 1. Equations (6) and (7) were used for the SNR calculation in the simulation. After the channel gain coefficients are generated, the noise power associated with the received signal power is generated for a particular SNR. A $\Delta f$ value of $10^{-5}$ was adopted in the simulation. The results of 5000 Monte Carlo simulations were averaged for each SNR.

For the iterative mode, the normalized CFOs were randomly selected independently from the random variable and were uniformly distributed over $[-0.5,0.5]$. The

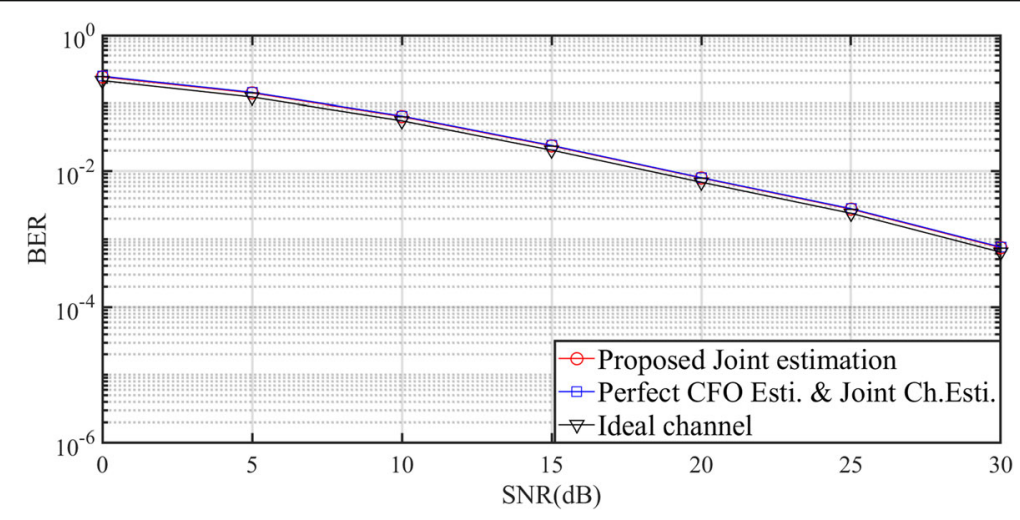

Fig. 8 The BER performance comparison for the joint estimation 


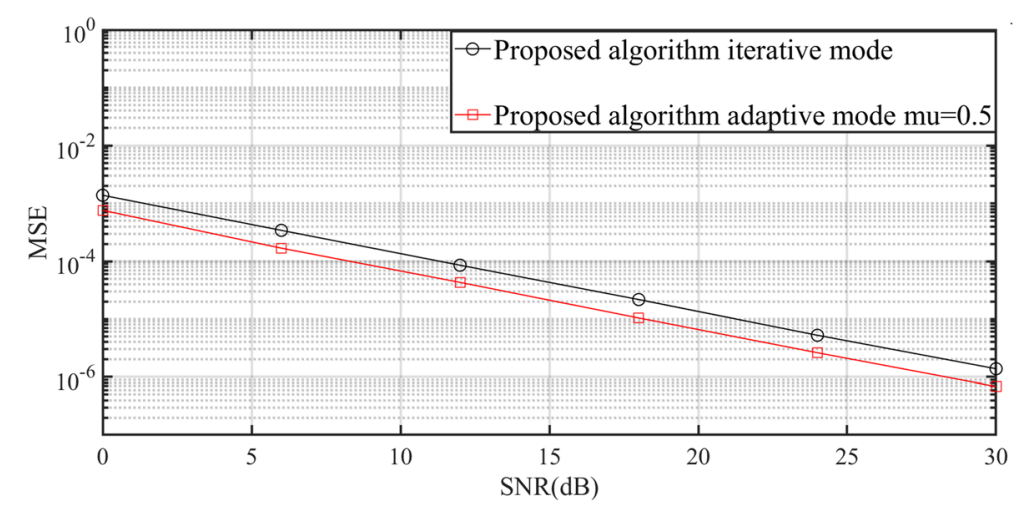

Fig. 9 The MSE performance for CFO estimation in the adaptive mode at $v=0 \mathrm{~km} / \mathrm{h}$

coefficients of the CIR were complex valued and generated independently from normally distributed random numbers.

Regarding the adaptive tracking mode, the coefficients of the CIR were generated using Jakes' model, with the carrier frequency equaling $3.5 \mathrm{GHz}$, which is to be adopted in 5G. Various velocities $(v)$ produced a time-varying Doppler shift. The channel variations in Jakes' model rely on the parameter of the maximum Doppler shift, which is determined by the carrier frequency and the mobile speed. Therefore, the change of the carrier frequency to scrutinize other frequencies in the $5 \mathrm{G}$ band is equivalent to the change of the mobile speed with a larger value for the specified carrier frequency of 3.5 GHz. Simulations were performed for various mobile speeds.

In the case of channel estimation errors, $\mathrm{E}\left(\|\hat{\mathbf{h}}-\mathbf{h}\|^{2}\right)$ was calculated and plotted using the sample averages. To obtain benchmarks for the performance evaluation, the lower bounds for the CFO and CIR in the iterative mode were derived using the Fisher information matrix based on (4), as in [31].

$$
\begin{aligned}
& {\left[\begin{array}{c}
\mathbf{r}_{1} \\
\vdots \\
\mathbf{r}_{M}
\end{array}\right]_{M \times 1}=\left[\begin{array}{ccc}
\mathbf{D}(\varepsilon) \mathbf{X} & & \mathbf{O} \\
& \ddots & \\
\mathbf{O} & & \mathbf{D}(\varepsilon) \mathbf{X}
\end{array}\right]_{M \times M}\left[\begin{array}{c}
\mathbf{h}_{1} \\
\vdots \\
\mathbf{h}_{M}
\end{array}\right]_{M \times 1}+\left[\begin{array}{c}
\mathbf{v}_{1} \\
\vdots \\
\mathbf{v}_{M}
\end{array}\right]_{M \times 1}} \\
& \Rightarrow \mathbf{r}=\left(\mathbf{I}_{M} \otimes \mathbf{D}(\varepsilon) \mathbf{X}\right) \mathbf{h}+\mathbf{v}
\end{aligned}
$$

where $\mathbf{r}=\left[\mathbf{r}_{1}, \ldots, \mathbf{r}_{M}\right]^{\mathrm{T}}, \mathbf{h}=\left[\mathbf{h}_{1}, \ldots, \mathbf{h}_{M}\right]^{\mathrm{T}}$

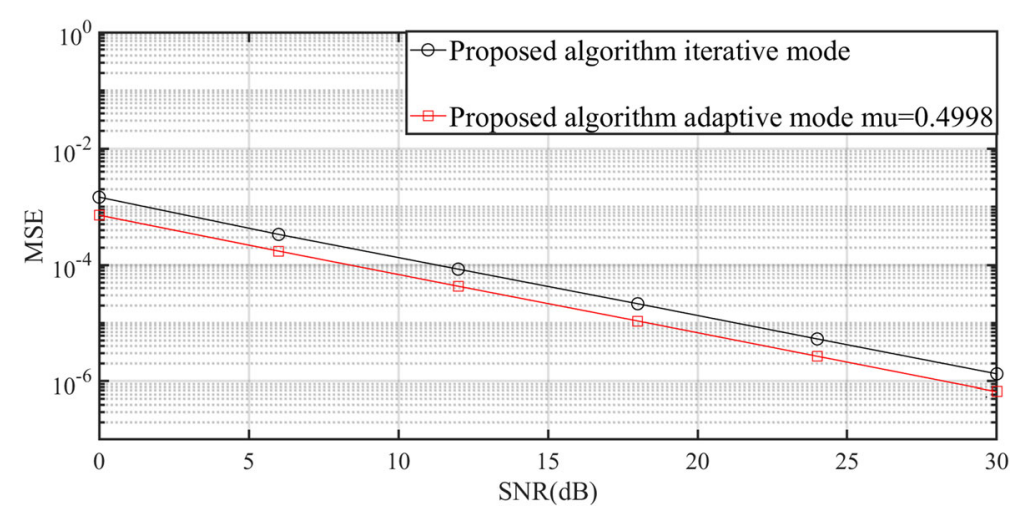

Fig. 10 The MSE performance for CFO estimation in the adaptive mode at $v=60 \mathrm{~km} / \mathrm{h}$ 


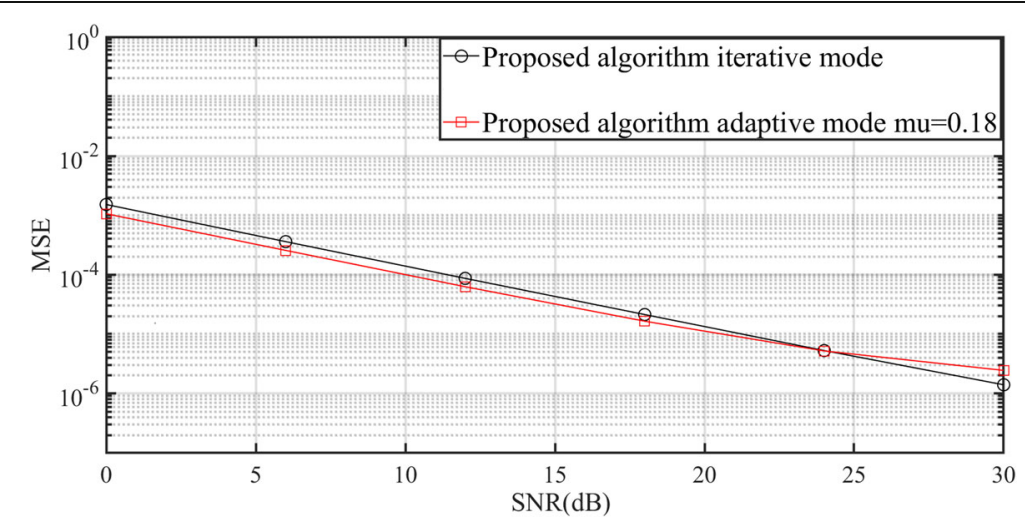

Fig. 11 The MSE performance for CFO estimation in the adaptive mode at $v=500 \mathrm{~km} / \mathrm{h}$

$$
\mathrm{CRB}(\varepsilon)=\frac{K^{2} \sigma_{\mathbf{v}}^{2}}{8 \pi^{2}}\left[\mathbf{h}^{H}\left[\mathbf{I}_{M} \otimes \mathbf{X}^{H} \mathbf{M} \Pi^{\perp} \mathbf{M X}\right] \mathbf{h}\right]^{-1}
$$

where $\otimes$ denotes the Kronecker product. Moreover, the following equation is obtained:

$$
\begin{aligned}
& \boldsymbol{\Pi}^{\perp}=\mathbf{I}_{K}-\mathbf{X}\left(\mathbf{X}^{H} \mathbf{X}\right)^{-1} \mathbf{X}^{H} \\
& \mathbf{M}=\operatorname{diag}(0,1,2, \cdots, K-1)
\end{aligned}
$$

Similarly, the CRB was obtained for the estimated $\hat{\mathbf{h}}$ as follows:

$$
\begin{gathered}
\mathrm{E}\left(\|\hat{\mathbf{h}}-\mathbf{h}\|^{2}\right)=\mathrm{E}\left[(\hat{\mathbf{h}}-\mathbf{h})(\hat{\mathbf{h}}-\mathbf{h})^{\mathrm{H}}\right] \geq \frac{\sigma_{\mathbf{v}}^{2}}{2}\left(2 \lambda+\gamma^{-1} \beta \beta^{\mathrm{H}}\right) \\
=C R B(\mathbf{h})
\end{gathered}
$$

where

Table 4 The parameter $\mu$ for other various mobile speeds

\begin{tabular}{ll}
\hline Mobile speed $\boldsymbol{v}(\mathbf{k m} / \mathbf{h})$ & Parameter $\boldsymbol{\mu}$ \\
\hline 0 & 0.5 \\
60 & 0.49980 \\
100 & 0.49967 \\
120 & 0.49955 \\
150 & 0.49800 \\
200 & 0.48 \\
300 & 0.41 \\
350 & 0.30 \\
400 & 0.22 \\
450 & 0.20 \\
500 & 0.18 \\
550 & 0.15 \\
600 & 0.12 \\
650 & 0.10 \\
700 & 0.06 \\
\hline
\end{tabular}




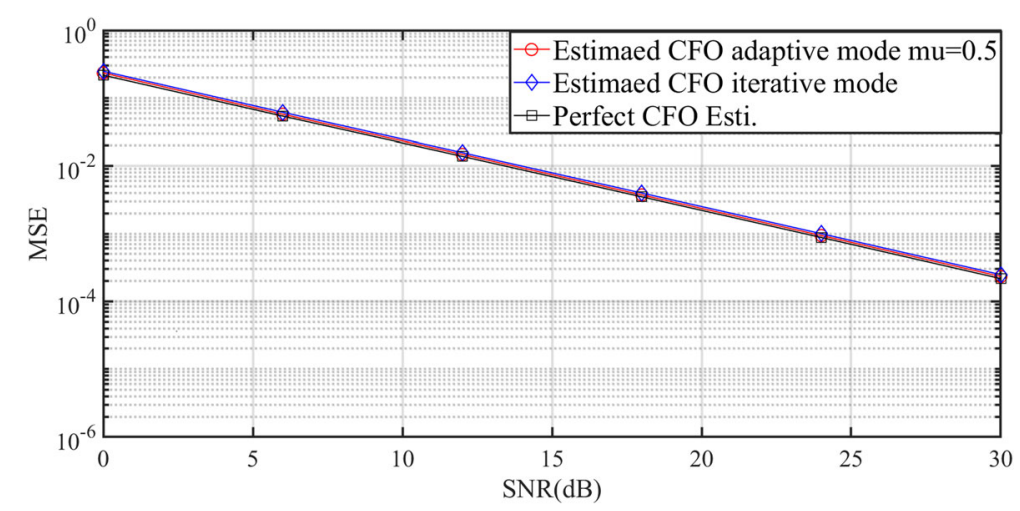

Fig. 12 The MSE performance for channel estimation in the adaptive mode at $v=0 \mathrm{~km} / \mathrm{h}$

$$
\begin{aligned}
& \lambda=\mathbf{I}_{M} \otimes\left(\mathbf{X}^{\mathrm{H}} \mathbf{X}\right)^{-1} \\
& \beta=\mathbf{I}_{M} \otimes\left[\left(\mathbf{X}^{\mathrm{H}} \mathbf{X}\right)^{-1} \mathbf{X}^{\mathrm{H}} \mathbf{D}(\varepsilon) \mathbf{X}\right] \mathbf{h} \\
& \gamma=\mathbf{h}^{\mathrm{H}} \mathbf{I}_{M} \otimes\left[\mathbf{X}^{H} \mathbf{M} \boldsymbol{\Pi}^{\perp} \mathbf{M} \mathbf{X}\right] \mathbf{h}
\end{aligned}
$$

\subsection{Algorithm performance in the iterative mode}

The mean squared error (MSE) performance of the proposed algorithm for CFO estimation in the iterative mode at different SNRs is revealed in Fig. 6. The performance of the proposed algorithm in the iterative mode was satisfactory and close to the CRB [refer to (26)].

The MSE performance of the proposed algorithm for channel estimation at different SNRs is illustrated in Fig. 7. Comparisons of the CRB [refer to (28)] and channel estimate with the estimates in the "perfect CFO estimation" condition, in which the CFOs are perfectly known, indicated that the proposed joint estimation algorithm provided satisfactory channel estimation performance.

In Fig. 8, the bit error rates (BERs) of the three schemes are illustrated as follows: (a) the "ideal channel" condition, which has perfect channel estimation and perfect CFO synchronization; (b) the "perfect CFO estimation with joint channel estimation" condition, in which CFOs are assumed to be known for estimating the CIR; and (c)

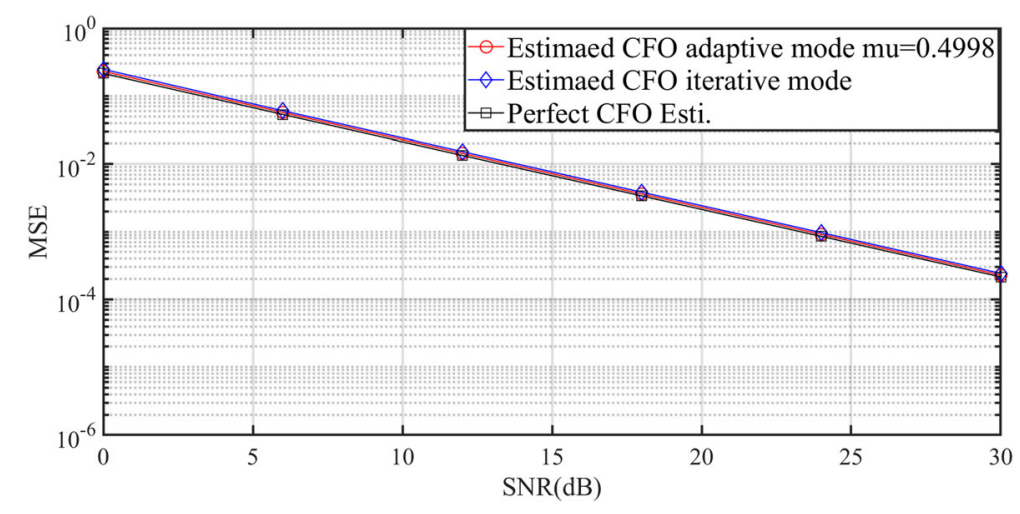

Fig. 13 The MSE performance for channel estimation in the adaptive mode at $v=60 \mathrm{~km} / \mathrm{h}$ 


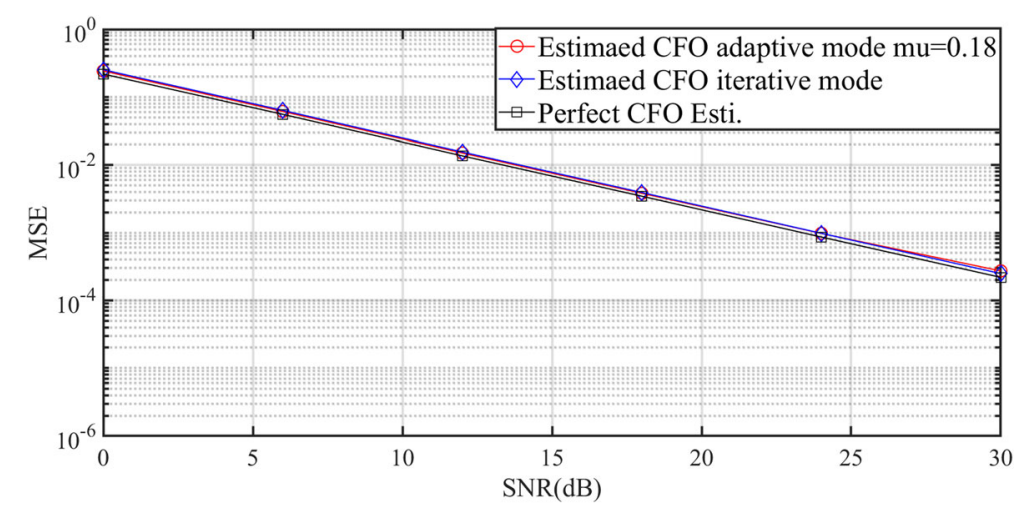

Fig. 14 The MSE performance for channel estimation in the adaptive mode at $v=500 \mathrm{~km} / \mathrm{h}$

"proposed joint CFO and channel estimation." The results indicate that the BER performance of the joint estimation is nearly identical to that with ideal assumptions. The proposed algorithm not only has a considerably lower computational complexity than the grid search-based method but also provides satisfactory performance, as revealed in Figs. 6, 7, and 8.

\subsection{Algorithm performance in the adaptive tracking mode}

The MSE performance of the proposed algorithm for CFO estimation in the adaptive mode at different SNRs is illustrated in Figs. 9, 10, and 11. To obtain intensive simulation results and the best MSE performance, the value of $\mu$ was selected as follows for various mobile speeds: (a) $\mu=0.5$ at $v=0 \mathrm{~km} / \mathrm{h}$ in Fig. 9, (b) $\mu=0.4998$ at $v=60 \mathrm{~km} / \mathrm{h}$ (the speed limit for automobiles in suburban areas) in Fig. 10, and (c) $\mu=0.18$ at $v=$ $500 \mathrm{~km} / \mathrm{h}$ (the average speed of high-speed rail, as defined for $5 \mathrm{G}$ ) in Fig. 11. The $\mu$ values for various other mobile speeds are presented in Table 4. The adaptive mode was executed on a per frame basis. The frame duration $t$ was $1 \mathrm{~ms}$. As expected, the simulation results indicated that the coefficient $\mu$ should be decreased when the velocity $v$ increases. The estimated CFO $\tilde{\varepsilon}_{T=t+\Delta t}$ of the next frame should have more weight than the estimated CFO $\tilde{\varepsilon}_{T=t}$ when the time variation is faster. Therefore, the weighting of the parameterized equation [refer to (23)] tends toward the second term.

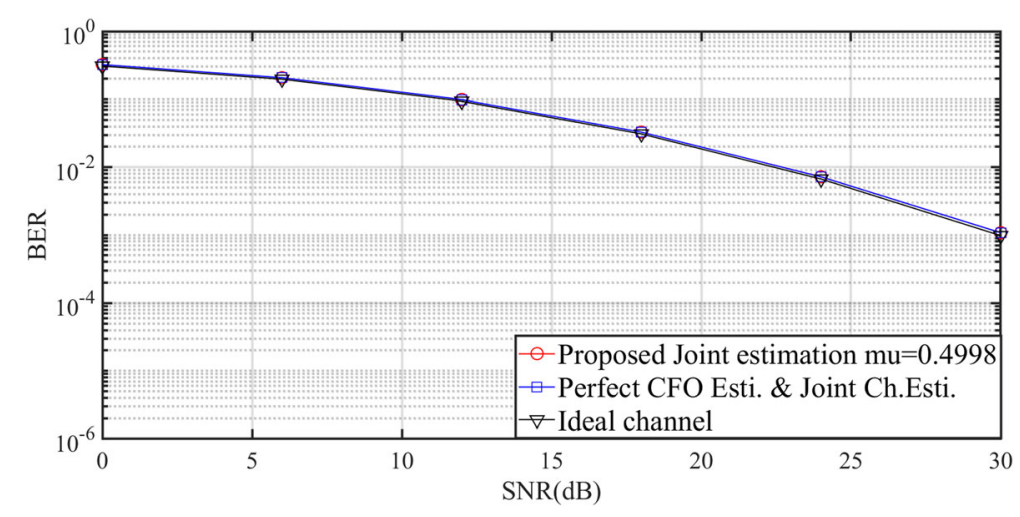

Fig. 15 The BER performance of the joint estimation in the adaptive mode at $v=60 \mathrm{~km} / \mathrm{h}$ 


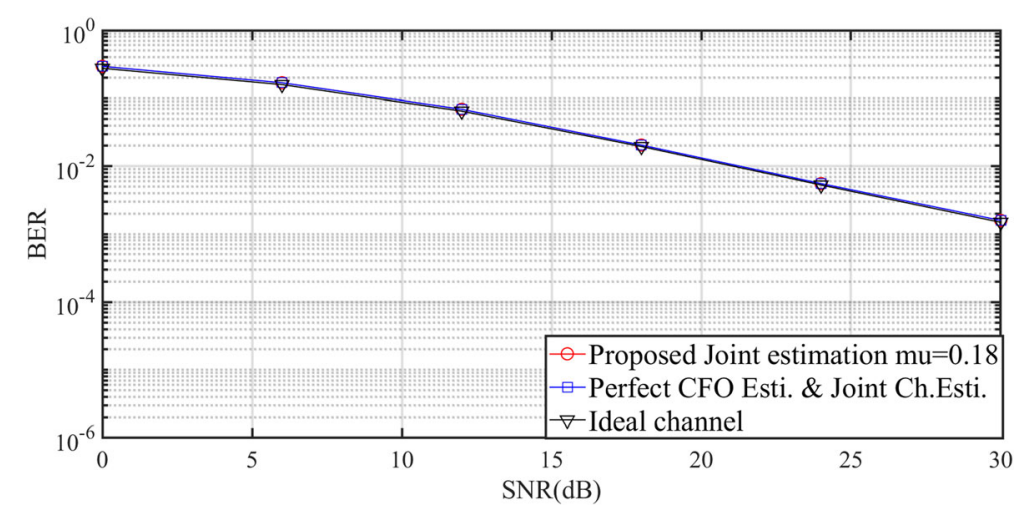

Fig. 16 The BER performance of the joint estimation in the adaptive mode at $v=500 \mathrm{~km} / \mathrm{h}$

As illustrated in Figs. 9 and 10, the performance of the proposed algorithm in the adaptive mode was superior to its performance in the iterative mode. As depicted in Fig. 11, the MSE performance of the proposed adaptive mode began to degrade at SNR $=24 \mathrm{~dB}$ due to high time variations, and $\mu$ was approximately selected. This phenomenon did not occur at low mobile speeds.

The MSE performance of the proposed algorithm for channel estimation in the adaptive mode is displayed in Figs. 12, 13, and 14. The value of the parameter $\mu$ is selected for various mobile speeds as: (a) $\mu=0.5$ at $v=0 \mathrm{~km} / \mathrm{h}$ in Fig. 12, (b) $\mu=0.4998$ at $v=$ $60 \mathrm{~km} / \mathrm{h}$ in Fig. 13, and (c) $\mu=0.18$ at $v=500 \mathrm{~km} / \mathrm{h}$ in Fig. 14, respectively, for the approximate best MSE performance. The results obtained with the proposed algorithm in the iteration mode and those obtained in the "perfect CFO estimation" condition were compared. The simulation results revealed that the performance obtained in the proposed adaptive mode was competitive with that obtained in the "perfect CFO estimation" condition (Figs. 9, 10, and 11).

Figures 15 and 16 display the BERs of the following three schemes: (1) the "ideal channel" condition, in which perfect channel estimation and CFO estimation are assumed; (2) the "perfect CFO estimation and joint channel estimation" condition, in which the CFOs are assumed to be known to estimate the CIR; and (3) the "proposed joint CFO and channel estimation" in the adaptive mode. To obtain the best BER performance, the values of $\mu$ were selected as follows: (a) $\mu=0.4998$ at $v=60 \mathrm{~km} / \mathrm{h}$ in Fig.

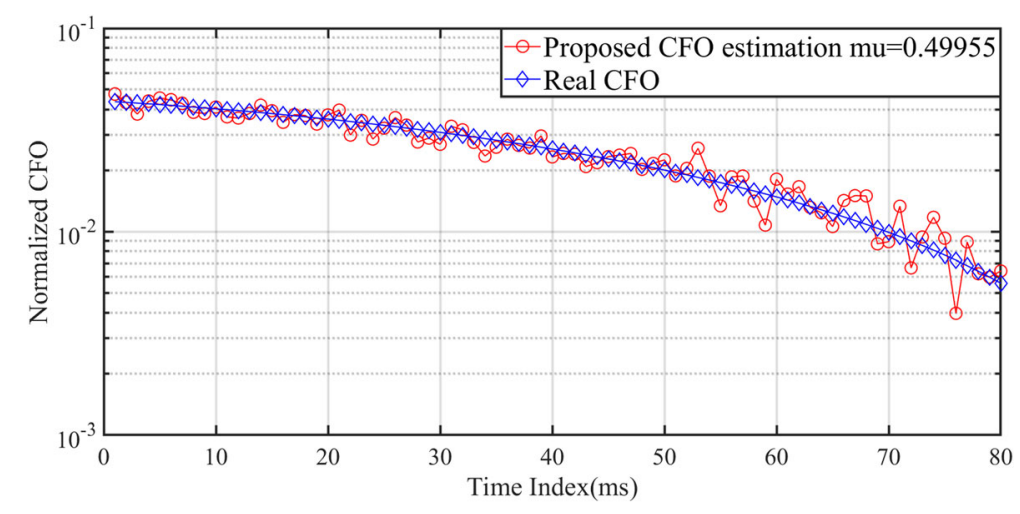

Fig. 17 Normalized real CFO vs. the estimated CFO for the time-varying scenario 


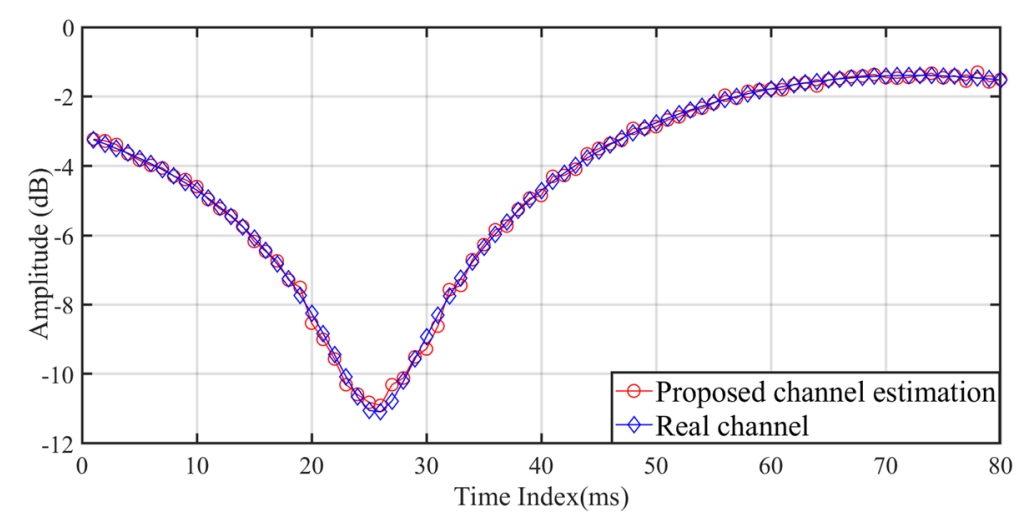

Fig. 18 Channels tracking performance for the time-varying scenario

15 and (b) $\mu=0.18$ at $v=500 \mathrm{~km} / \mathrm{h}$ in Fig. 16. The results indicated that the proposed adaptive mode estimation offers a BER performance nearly identical to that obtained with ideal assumptions.

The tracking ability of the proposed adaptive mode was examined at $v=120 \mathrm{~km} / \mathrm{h}$ (Fig. 17). The estimate was compared with a real time-varying CFO. The comparison indicated that the proposed algorithm can track a time-varying CFO in the adaptive mode. For a vehicle velocity of $120 \mathrm{~km} / \mathrm{h}$, the tracking results of the $\mathbf{h}_{11}$ are plotted in Fig. 18, where we select one of the channel gains in the illustration. The time variations of the channel were accurately tracked.

\section{Conclusion}

In this paper, we propose ML-based algorithms for joint CFO and channel estimation. The proposed methods provided fairly competitive performance to that of CRBs. Moreover, the proposed methods have a lower computational complexity than the grid search-based method does. In addition, an adaptive mode is proposed to improve the algorithm performance. The adaptive method is used to obtain the weighted CFO; thus, the adaptive mode can enhance the performance of the original proposed algorithm and provide tracking capability for time-varying parameters. The parameter $\mu$ should be adjusted according to operational environments.

\section{Abbreviations}

CFO: Carrier frequency offset; MIMO-OFDM: Multiple input multiple output-orthogonal frequency-division multiplexing; MSE: Mean squared error; CRB: Cramer-Rao lower bound; 5G: The fifth-generation wireless networks; ISI: Inter-symbol interference; CP: Cyclic prefix; ML: Maximum likelihood; CIR: Channel impulse response; TX: Transmit; RX: Receive; IDFT: Inverse discrete Fourier transform; DFT: Discrete Fourier transform; SNR: Signal-to-noise ratio; BER: Bit error rate

\section{Acknowledgements}

The authors would like to thank the editors and anonymous reviewers for their constructive comments and suggestions, which helped improve the manuscript. This work was supported in part by the Ministry of Science and Technology, Taiwan, under Grant MOST 108 - 2221 - E - 008 - 020 - MY2 and in part by the Qualcomm Technologies, Inc., USA, under Grant No. SOW NAT-435657.

\section{Authors' contributions}

The algorithms proposed in this paper have been conceived by N-H Cheng, K-C Huang, Y-F Chen, and S-M Tseng. Y-F Chen designed the experiments. N-H Cheng and K-C Huang performed the experiments and analyzed the results. N-H Cheng, $\mathrm{K}-\mathrm{C}$ Huang, and Y-F Chen wrote the paper. The authors read and approved the final manuscript. 
of Communication Engineering at National Central University and co-advised by the Department of Electronic Engineering at Chang Gung University. His current research areas include signal processing algorithm designs for wireless communication systems and ESD protection of HEMT devices.

Kai-Chieh Huang received his BS degree at the department of communication engineering from Yuan Ze University in 2016 and then got MS degree in communication engineering from National Central University in 2018. The area of his current research is about digital signal processing and algorithm designs for wireless communication systems.

Yung-Fang Chen ( $\left.S^{\prime} 95-M^{\prime} 98\right)$ received his BS degree in computer science and information engineering from National Taiwan University, Taipei, Taiwan, in 1990; received his MS degree in electrical engineering from the University of Maryland, College Park, in 1994; and obtained a PhD in electrical engineering from Purdue University, West Lafayette, IN, in 1998. From 1998 to 2000, he worked with the CDMA Radio Technology Performance Group in Lucent Technologies, Whippany, NJ. Since 2000, he has been a Professor in the Department of Communication Engineering, National Central University, Taoyuan, Taiwan. His research interests include resource management algorithm designs for communication systems and signal processing algorithm designs for wireless communication systems. Shu-Ming Tseng received the B.S. degree from National Tsing Hua University (highest honors), Taiwan, and the M.S. and Ph.D. degrees from Purdue University, IN, USA, all in electrical engineering, in 1994, 1995, and 1999, respectively. He was with the Department of Electrical Engineering, Chang Gung University, Taiwan, from 1999 to 2001. Since 2001, he has been with the Department of Electronic Engineering, National Taipei University of Technology, Taiwan, where he has been a Professor since 2007. His research interests are MUMIMO, OFDMA, cross layer optimization for video transmission, jamming resiliency, NOMA for 5G, and network performance evaluation. He has published $39 \mathrm{SCl}$ journal papers. He has served as an Editor for KSII Transactions on Internet and Information Systems, indexed in SCI, since 2013. He was on sabbatical and a visiting scholar with Department of Electrical and Computer Engineering, UC San Diego, USA, in the 2014-2015 academic year. He is listed in Marquis Who's Who in World since 2006.

\section{Funding}

This work was supported in part by the Ministry of Science and Technology, Taiwan, under Grant MOST 108 - 2221 - E - 008 - 020 - MY2 and in part by the Qualcomm Technologies, Inc., USA, under grant No. SOW NAT-435657.

\section{Availability of data and materials}

The datasets generated during the current study are not publicly available but are available from the corresponding author on reasonable request.

\section{Ethics approval and consent to participate}

All data and procedures performed in this paper were in accordance with the ethical standards of research community. This paper does not contain any studies with human participants or animals performed by any of the authors.

\section{Competing interests}

The authors declare that they have no competing interests.

\section{Author details}

'Department of Communication Engineering, National Central University, Jungli City, Taoyuan, Taiwan, Republic of China. ${ }^{2}$ Department of Electronic Engineering, National Taipei University of Technology, Taipei, Taiwan, Republic of China.

Received: 27 August 2019 Accepted: 11 December 2020

Published online: 13 January 2021

\section{References}

1. T. Mao, Q. Wang, Z. Wang, S. Chen, Novel index modulation techniques: A survey. IEEE Commun. Surv. Tutor. 21, 315$348(2019)$

2. Y. Liu, X. Zhao, H. Zhou, Y. Zhang, T. Qiu, Low-complexity spectrum sensing for MIMO communication systems based on cyclostationarity. EURASIP J. Adv. Signal. Process. 2019, article id 29 (2019)

3. Y. Sheng, Z. Tan, G. Li, Single-Carrier Modulation with ML Equalization for Large-Scale Antenna Systems over Rician Fading Channels (IEEE International Conference on Acoustics, Speech and Signal Processing (ICASSP), Florence, 2014)

4. L. Nasraoui, L.N. Atallah, M. Siala, Robust Synchronization Approach for MIMO-OFDM Systems with Space-Time Diversity (IEEE 81st Vehicular Technology Conference (VTC Spring), Glasgow, 2015)

5. A. Diliyanzah, R.P. Astuti, B. Syihabuddin, Dynamic CFO Reduction in Various Mobilities Based on Extended Kalman Filter for Broadband Wireless Access Technology (International Conference on Information Technology and Electrical Engineering (ICITEE) Conference, Yogyakarta, 2014)

6. A. Jhingan, L. Kansal, G.S. Gaba, F. Tubbal, S. Abulgasem, Performance Analysis of OFDM System Augmented with SC Diversity Combining Technique in Presence of CFO (12th International Conference on Telecommunication Systems, Services, and Applications (TSSA), Yogyakarta, 2018)

7. M.-O. Pun, M. Morelli, C.-C. Jay Kuo, Iterative detection and frequency synchronization for OFDMA uplink transmissions. IEEE Trans. Wirel. Commun. 6, 629-639 (2007)

8. G. Li, T. Li, M. Xu, X. Zha, Y. Xie, Sparse massive MIMO-OFDM channel estimation based on compressed sensing over frequency offset environment. EURASIP J. Adv. Signal. Process. 2019, article id 31 (2019). https://doi.org/10.1186/s13634019-0627-3

9. D. Bai, W. Nam, J. Lee, I. Kang, Comments on: "a technique for orthogonal frequency division multiplexing frequency offset correction". IEEE Trans. Commun. 61, 2109-2111 (2013)

10. A.J. Coulson, Maximum likelihood synchronization for OFDM using a pilot symbol: Algorithm. IEEE J. Sel. Areas. Commun. 19, 2486-2494 (2001) 
11. J.J. van de Beek, O. Edfors, M. Sandell, On channel estimation in OFDM systems. Proc. Veh. Technol. Conf. 2, 815-819 (1995)

12. M. Morelli, U. Mengali, A comparison of pilot-aided channel estimation methods for OFDM systems. IEEE Trans. Signal. Proc. 49, 3065-3073 (2001)

13. B.H. Fleury, M. Tschudin, R. Heddergott, D. Dahlhaus, K.I. Pedersen, Channel parameter estimation in mobile radio environments using the SAGE algorithm. IEEE J. Sel. Areas. Commun. 17, 434-450 (1999)

14. V.S. Hendre, M. Murugan, R.V. Jawale, Channel Residual Energy Based Combine Estimation of Imperfections of Receiver in MIMO-OFDM System (International Conference on Pervasive Computing (ICPC), Pune, 2015)

15. F. Yang, P. Cai, H. Qian, X. Luo, Pilot contamination in massive MIMO induced by timing and frequency errors. IEEE Trans. Wirel. Commun. 17, 4477-4492 (2018)

16. A.K. Dutta, K.V.S. Hari, L. Hanzo, Minimum-error-probability CFO estimation for multiuser MIMO-OFDM systems. IEEE Trans. Veh. Technol. 64, 2804-2818 (2015)

17. S.H. Aswini, B.N.A. Lekshmi, S. Sekhar, S.S. Pillai, MIMO-OFDM Frequency Offset Estimation for Rayleigh Fading Channels (First International Conference on Computational Systems and Communications (ICCSC), Trivandrum, 2014)

18. M.O. Pun, M. Morelli, CCJ, Kuo, maximum-likelihood synchronization and channel estimation for OFDMA uplink transmissions. IEEE Trans. Commun. 54, 726-736 (2006)

19. I. Ziskind, M. Wax, Maximum likelihood localization of multiple sources by alternating projection. IEEE Trans. Acoust. Speech Signal Process. 36, 1553-1560 (1998)

20. A. Saemi, V. Meghdadi, J.P. Cances, M.R. Zahabi, J.M. Dumas, ML Time-Frequency Synchronization for MIMO-OFDM Systems in Unknown Frequency Selective Fading Channels (Proceedings of the IEEE International Symposium on Personal, Indoor and Mobile Radio Commun. (PIMRC), Helsinki, 2007), pp. 1-5

21. S. Salari, M. Ahmadian, M. Ardebilipour, J.P. Cances, V. Meghdadi, EM-based turbo receiver design for low-density paritycheck-coded MIMO-OFDM systems with carrier-frequency offset. IET Commun. 2, 107-112 (2008)

22. X. Chen, A. Wolfgang, A. Zaidi, MIMO-OFDM for Small Cell Backhaul in the Presence of Synchronization Errors and Phase Noise (IEEE International Conference on Communications Workshops (ICC Workshops), Paris, 2017)

23. B. Zhou, Q. Chen, F. Shen, Q. Ci, On the Joint Carrier Frequency Offset Estimation and Channel Tracking Limits for MIMOOFDM System over High-Mobility Scenarios (IEEE International Conference on Acoustics, Speech and Signal Processing (ICASSP), Nanjing, 2016)

24. S. Salari, M. Ardebilipour, M. Ahmadian, Joint maximum-likelihood frequency offset and channel estimation for multipleinput multiple-output-orthogonal frequency-division multiplexing systems. IET Commun. 2, 1069-1076 (2008)

25. S. Salari, M. Ahmadian, M. Ardebilipour, V. Meghdadi, J.P. Cances, Maximum-Likelihood Carrier-Frequency Synchronization and Channel Estimation for MIMO-OFDM Systems (2007 Wireless Telecommunications Symposium, Pomona, 2007), pp. 1-5

26. S. Salari, M. Heydarzadeh, J.P. Cances, Joint Maximum-Likelihood Frequency Synchronization and Channel Estimation in MIMO-OFDM Systems with Timing Ambiguity (International Symposium on Wireless Communication Systems (ISWCS), Paris, 2012), pp. 954-958

27. S.H. Chiu, K.C. Fu, Y.F. Chen, A Modified Algorithm for Joint Frequency Offset and Channel Estimation in OFDM Systems (International Conference on Communications in China (ICCC), Xi'an, 2013)

28. M. Morelli, U. Mengali, Carrier-frequency estimation for transmissions over selective channels. IEEE Trans. Commun. 48, $1580-1589$ (2000)

29. S. Ohno, E. Manasseh, M. Nakamoto, Preamble and pilot symbol design for channel estimation in OFDM systems with null subcarriers. EURASIP J. Wirel. Commun. Netw. 2011, article no. 2 (2011)

30. H. Hojatian, M.J. Omidi, H. Saeedi-Sourck, A. Farhang, Joint CFO and Channel Estimation in OFDM-Based Massive MIMO Systems (International Symposium on Telecommunications (IST), Tehran, 2016)

31. P. Ciblat, P. Bianchi, M. Ghogho, Training sequence optimization for joint channel and frequency offset estimation. IEEE Trans. Signal Process. 56, 3424-3436 (2008)

\section{Publisher's Note}

Springer Nature remains neutral with regard to jurisdictional claims in published maps and institutional affiliations.

\section{Submit your manuscript to a SpringerOpen ${ }^{\circ}$ journal and benefit from:}

- Convenient online submission

- Rigorous peer review

- Open access: articles freely available online

- High visibility within the field

- Retaining the copyright to your article

Submit your next manuscript at $\boldsymbol{s p r i n g e r o p e n . c o m ~}$ 